\title{
La maternité sociale et le Mouvement Populaire des Familles durant les Trente Glorieuses
}

\section{Geneviève Dermenjian et Dominique LOISEAU}

\section{(2) OpenEdition \\ 1 Journals}

\section{Édition électronique}

URL : https://journals.openedition.org/clio/1449

DOI : $10.4000 /$ clio. 1449

ISSN : 1777-5299

Éditeur

Belin

Édition imprimée

Date de publication : 1 avril 2005

Pagination : 91-105

ISBN : 2-85816-781-8

ISSN : 1252-7017

Référence électronique

Geneviève Dermenjian et Dominique LOISEAU, «La maternité sociale et le Mouvement Populaire des Familles durant les Trente Glorieuses », Clio. Histoire, femmes et sociétés [En ligne], 21 | 2005, mis en ligne le 01 juin 2007, consulté le 24 avril 2022. URL : http://journals.openedition.org/clio/1449 ; DOI : https://doi.org/10.4000/clio.1449

Ce document a été généré automatiquement le 24 avril 2022.

Tous droits réservés 


\title{
La maternité sociale et le Mouvement Populaire des Familles durant les Trente Glorieuses
}

\author{
Geneviève Dermenjian et Dominique LOISEAU
}

1 Les tâches de la mère au foyer ont été et demeurent multiples. Cette maternité « privée " peut s'étendre, ou faire place à d'autres types de maternités exercées par des femmes ${ }^{1}$ issues de milieux favorisés ou plus modestes qui suppléent, individuellement ou collectivement, aux manques matériels et moraux des familles les plus démunies. "Sous le terme de "maternité sociale", écrit Michelle Perrot à propos du XIX" siècle, on assiste à une véritable mobilisation féminine dans tout l'occident $»^{2}$.

2 Cette "maternité sociale ${ }^{3}$ " a pris dans un premier temps une forme charitable, dans un grand nombre d'organisations à caractère religieux puis social. Pensons à MarieAntoinette créant à la fin du XVIII siècle la Société de charité maternelle, ou à la mise en place des salles d'asiles au XIX ${ }^{e}$ siècle, ou encore à l'activité des religieuses infirmières et enseignantes. Cette forme charitable de la maternité sociale peut coexister avec une maternité réelle, mais aussi avec un dépassement de cette maternité réelle au profit d'une maternité virtuelle qui assure, hors du foyer, les fonctions liées au "rôle maternel ». Elle ne se confond alors ni avec les mères biologiques ni avec les mères spirituelles ${ }^{4}$ et oriente en priorité ses interventions vers les mères et les enfants, éléments fondamentaux et fragiles de la famille et de la société du XIXe siècle. Elle prend au fil du temps un caractère plus complexe et plus élaboré, marqué par exemple par un encadrement prolongé des personnes assistées, par des enquêtes concernant les familles bénéficiaires, par la naissance de nouvelles professions.

$3 \mathrm{Au} \mathrm{XX}$ siècle, des groupements à caractère politique, religieux ou social, déploient eux aussi des activités sociales variées. Ainsi, la mouvance catholique sociale a donné naissance, dans le cadre de l'Action catholique spécialisée, à des associations qui se donnaient pour fonction la formation et la promotion d'un milieu social donné, grâce aux efforts de ce même milieu se prenant lui-même en charge. Parmi ces associations, la mouvance du Mouvement populaire des familles (MPF) a réuni, des années 1940 à la 
fin des Trente Glorieuses, un grand nombre de foyers ouvriers militants. La description de ses activités et une rapide comparaison avec la mouvance communiste indiquent ici l'originalité et les limites de cette maternité sociale.

Une maternité sociale pratiquée " de l'intérieur "

4 L'Action catholique est encouragée dans l'entre-deux-guerres par le pape Pie XI. Celuici propose aux laïcs la « promotion du laïcat ${ }^{5}$ » qui consiste à prendre une plus grande place dans l'Église et la société. Le MPF s'inscrit au départ dans les "mouvements » issus de la matrice Action catholique spécialisée et forme la branche adulte de la JOCJOCF. Créé en 1933-1935 sous le nom de Ligue ouvrière chrétienne, le MPF (1941) est un mouvement de foyers, innovation dans le monde catholique et militant. Il se déconfessionnalise en 1949 et donne naissance, à la suite d'une scission survenue en 1951, à deux mouvements, chacun gérant les associations de quartier ${ }^{6}$ déjà existantes et en créant de nouvelles. Il s'agit du Mouvement de libération du peuple (MLP) et de la Confédération syndicale des familles (CSF) d'une part, du Mouvement de libération ouvrière (MLO) et de l'Association populaire familiale (APF) d'autre part. La règle de la mixité, effective de la décennie quarante à la décennie soixante, y est de moins en moins une réalité ensuite, car le taux de féminisation augmente sans cesse, pour atteindre au moins $80 \%$ dans les années 1990.

5 Malgré la scission, et sauf cas particulier, nous employons l'expression « mouvance MPF » pour caractériser l'ensemble de ces organisations, même déconfessionnalisées et ouvertes à des militants de tous horizons, car elles présentent de nombreux points communs. Par ailleurs, dissocier à l'extrême rendrait le propos peu intelligible dans le cadre restreint de cet article.

6 Les organisations de la mouvance MPF se développent durant les 30 Glorieuses dans les quartiers populaires, notamment autour de revendications liées au quotidien des familles, du soutien aux luttes ouvrières ${ }^{7}$ et de la mise en place de "services » qui représentent la tendance maternité sociale de la mouvance. Les mères de famille de l'organisation sont d'abord mères au foyer de leurs propres enfants, pour lesquels elles font tout le nécessaire, mais elles sont aussi militantes en tant que mères défendant dans le quartier leurs propres intérêts et ceux de toutes les mères ouvrières et de tous leurs enfants. Elles dépassent leur seul cas personnel pour englober celui des autres familles ouvrières, adoptant ainsi une première attitude et une première pratique qui relèvent de la maternité sociale. Avec cette nuance toutefois que la maternité sociale est généralement le fait de femmes de milieux étrangers au monde populaire démuni, alors que les militantes du MPF appartiennent à ce milieu et en partagent toutes les difficultés.

7 Le militantisme des mères au foyer du MPF trouve son explication dans l'émergence d'une nouvelle génération de femmes et d'hommes, le plus souvent formée par la JOCJOCF dès 1927-1928. Les militantes souhaitent ne pas en rester à la situation de leurs mères confinées au foyer. Elles veulent, selon leur expression, «profiter de la vie » et continuer le militantisme appris à la JOCF qui a constitué le socle de leur existence de jeunes filles et leur a transmis un ensemble de valeurs et de pratiques. Elles y ont appris notamment la valeur intrinsèque des jeunes travailleurs et l'égalité entre filles et garçons, une jeune travailleuse ayant mêmes droits et devoirs, et même voix dans les décisions qu'un jeune travailleur. Ceci reste vrai pour les femmes mariées, à qui tous les espaces de vie et de travail sont ouverts, avec toutefois une orientation privilégiée vers le foyer. 
8 Transposé dans la vie de couple, cela consiste pour les femmes mariées à s'investir de façon militante autrement que comme «accompagnatrice » du militantisme du mari. L'idéal d'engagement social des femmes s'est donc transmué, dans ce milieu, de la neutralité bienveillante ou du soutien matériel et moral du mari à une implication directe et spécifique, théoriquement de même valeur que celle du conjoint. Cette disposition reçoit l'approbation totale de l'organisation qui structure et légitime l'activité en faveur des mères et de la famille ouvrières. Mais, dans nombre de cas, et notamment à la base des organisations, c'est la volonté des femmes qui leur permet de faire passer ce militantisme dans la pratique, bouleversant ainsi les rapports sociaux de sexe au sein du couple.

9 Pour la mouvance MPF, l'engagement militant est en effet formateur et produit une « promotion » des individus et de la collectivité. La promotion recherchée des militants est inséparable de celle de l'ensemble de l'organisation et donc des femmes qui la composent - et de celle de l'ensemble du milieu populaire; d'où la nécessité d'un investissement militant en son sein. Ce milieu populaire, dont les militants et militantes font partie, doit se prendre en charge lui-même, selon la maxime : "entre eux, par eux, pour eux ». Objectivement, le territoire de la maternité sociale du MPF est pratiquement limité au monde ouvrier, mais celui-ci représente dans les années considérées 30 à $35 \%$ de la population active.

10 Notons la présence au sein de la mouvance MPF d'un grand nombre de célibataires bienvenues "puisque tout le monde, même les célibataires, a une famille ». Ces dernières jouent souvent le rôle de « mères sociales » et occupent une place importante dans les responsabilités et la représentation, état de fait qui se retrouve dans un grand nombre d'organisations à caractère social et bien au-delà, par exemple dans les syndicats féminins de la CFTC de l'entre-deux guerres. La maternité sociale n'est pas ici seulement affaire de personnes, en l'occurrence les mères. L'organisation elle-même peut être assimilée à une "mère sociale » face au milieu qu'elle représente, défend, incarne, notamment en raison des objectifs déjà évoqués de "promotion ${ }^{8}$ " et d'intégration de ses "enfants" dans une seule et même grande famille, où tous avancent en même temps. On est en présence d'un emboîtement de " maternités »: les militantes, elles-mêmes mères, sont les mères sociales des mères ouvrières et sont insérées dans la maternité sociale de l'organisation.

11 Ainsi, qu'elle soit individuelle, collective ou organisationnelle, la maternité sociale repose ici sur une décision prise en fonction de présupposés idéologiques divers (humanisme, charité, sens religieux ou civique...) proposés ou relayés par une organisation. Elle s'appuie sur un socle " doctrinal », alors que la maternité " réelle » est biologique au premier chef.

12 La mouvance communiste est elle aussi présente sur ce terrain de la maternité sociale, en particulier par le biais de l'Union des femmes françaises et des municipalités communistes. La maternité sociale de la mouvance communiste se distingue de celle de la mouvance MPF par son objectif global de « libération » des femmes dans le cadre de la lutte des classes et, malgré l'affirmation que « l'émancipation des travailleurs sera l'oeuvre des travailleurs eux-mêmes ", par une posture d'extériorité relative, liée à son statut auto-proclamé d'avant-garde. Ainsi, on peut lire dans L'Humanité que « Le parti communiste [se] penche avec sollicitude sur le sort des femmes de notre pays ${ }^{9}$ » et l'UFF, lors de son deuxième congrès (1947), indique que « c'est le souci permanent de l'UFF que de se pencher sur la peine des femmes ». Les organisations sont mères 
virtuelles du monde ouvrier avec tous les dangers que cela comporte, notamment celui de parler et agir à la place de "ses enfants». Le MPF refuse, quant à lui, de "se pencher " sur les problèmes de la classe ouvrière; son rejet à fleur de peau du paternalisme et son appartenance revendiquée à la classe ouvrière le fait se positionner comme les vivant avec elle de l'intérieur, sans aucun statut d'avant-garde, partageant les mêmes conditions de vie et les mêmes aspirations que les autres ouvriers. C'est d'ailleurs, sur le terrain, le cas de beaucoup de communistes, mais pas de leur parti en tant que tel.

13 Tout en soulignant les différences entre les mouvances catholique-sociale et communiste, il faut rappeler que la mouvance MPF (et le catholicisme-social) a aussi des objectifs politiques, au sens large du terme. Leurs prosélytismes respectifs les mettent d'ailleurs en concurrence au niveau de l'action de quartier. Concurrence, lutte d'influence qui, parfois, sur le terrain, n'empêchent pas une estime réciproque entre militantes.

La fonction « maternité sociale » au MPF

14 Comment atteindre l'objectif de cette maternité sociale où les mères de familles ouvrières investissent l'espace public en direction des autres mères de familles ouvrières pour les organiser, susciter leur "prise de conscience", enclencher une formation si minime soit-elle? Les trois objectifs déjà définis par la JOC-JOCF (école, service, corps représentatif) sont constamment évoquées par les militantes et l'organisation. Ainsi, sont créés des « services » multiples et efficaces dont certains sont au départ simplement destinés à soulager le travail des mères, à leur permettre de se reposer, d'avoir du temps libre, vu leur extrême fatigue ${ }^{10}$. Les premiers services sont nés pendant la Seconde Guerre mondiale, tel celui des femmes de prisonniers qui compte, selon sa présidente, 200000 adhérentes en 1944-1945. Ce service est mis en place dès la fin de 1940 pour aider les femmes à tenir bon face à l'accumulation des soucis matériels (problèmes financiers, alourdissement des tâches quotidiennes) et moraux (solitude, dépendance vis-à-vis de l'entourage), tout en sauvegardant les «droits des absents». Regroupées, formées, «les femmes de prisonniers peuvent dépasser leurs difficultés personnelles en aidant les autres femmes ou en s'occupant de l'association. Par là, elles se donnent aux autres, le don de soi étant alors reconnu comme une dimension essentielle de la personnalité féminine ${ }^{11}$ ». Par là également, les présidentes locales deviennent les « mamans » de leur association ${ }^{12}$.

15 La multiplicité des services est particulièrement visible après 1945 : groupements d'achats, aide à domicile, vestiaires, services ménagers. Parmi ceux-ci, on trouve les cireuses, les aspirateurs, les machines à coudre et à tricoter, les chargeurs de batterie, mais surtout les machines à laver, fleuron des services, qui commence à la fin des années 1940 et culmine au milieu des années 1960. De petites machines, achetées par l'association, sont réparties dans les quartiers ; une responsable y organise le calendrier de prêt et l'encaissement de la quote-part demandée aux usagères afin d'éviter l'assistanat. La gestion collective par les usagers, souhaitée par le MPF, n'est pas toujours réalisée, mais le service en tant que tel connaît un grand succès, en ces années où la lessive représente une tâche particulièrement lourde et fatigante.

16 L'ensemble de ces services est pour les militantes un outil destiné à favoriser la promotion des mères et des familles ouvrières. De ce point de vue, le service le plus pérennisé est celui des travailleuses familiales, qui ouvrit sur l'économie sociale. Il débute lui aussi à la fin des années 1940 mais existe toujours, sous une forme 
aujourd'hui institutionnalisée, avec des gestionnaires salariées qui ont remplacé les militantes de quartier. Par contre, dès le début, les travailleuses familiales sont des salariées, en général à plein temps. Ceci, outre l'existence d'un diplôme reconnu et qualifiant, en marque aujourd'hui encore la spécificité parmi les nombreuses formes d'aide à domicile qui se sont développées. Par ce service des travailleuses familiales, les mères de famille en difficulté reçoivent une aide matérielle, mais aussi des conseils pour la gestion globale des tâches à effectuer, le budget, les soins aux enfants. Le financement par les Caisses d'allocations familiales prouve, comme dans le cas de la formation ménagère mise en place par la mouvance MPF, la reconnaissance du service.

17 «Donner du confort » aux mères en allégeant le poids du travail ménager était aussi destiné à leur permettre de s'investir socialement, de venir aux réunions et donc de se former. Toute "prise de conscience " ou action militante des mères est considérée comme une "promotion» rejaillissant sur l'ensemble du monde ouvrier. De plus, la famille devait en être consolidée car les mères seraient de meilleures épouses et éducatrices. Les militantes soulignent à cet effet l'importance, de leur point de vue, d'une « formation ménagère » dont elles font remarquer l'inexistence quasi totale dans les milieux populaires fréquentés. Si elles en ont elles-mêmes reçu une, elles considèrent qu'elles ont « eu de la chance $»^{13}$. En situant les mères au coeur du processus éducatif, elles sont les héritières inconscientes et paradoxales des républicains du XIX siècle, qui donnaient aux mères républicaines la responsabilité... de former des républicains et donc d'arracher les enfants à l'influence de l'Église.

18 Là existent des différences notables avec la mouvance communiste. Au parti communiste, contrairement à ce qui se passe au MPF, l'apologie du militantisme et du militant "universel», mais de fait masculin, empêche d'appréhender les femmes comme différentes des hommes dans leur rapport au militantisme. Différentes non par leur « nature » de femmes, mais par leur «situation » de mères écrasées de travail, qui n'est pas prise en compte pour leur permettre de militer. Dans la pratique, le principe de l'égalité des sexes place les militantes dans une posture inégale par rapport à celle des militants. La structure communiste développe deux tendances, aussi excluantes l'une que l'autre : tu as des enfants donc tu ne peux pas militer ; tu as des enfants, on ne veut pas le savoir, tu milites comme les autres. Après 1945 apparaît une variante de la première formule : tu ne peux militer au PC, tu vas à l'UFF. Celle-ci, s'adressant dans les quartiers aux mêmes femmes que le MPF, est ainsi entachée du soupçon de «sousmilitantisme " puisque ne touchant pas au militantisme " noble ", syndicalisme, action politique, luttes ouvrières dans les usines... Ceci dit, la hiérarchie des lieux militants existe aussi au MPF, entre les associations de quartier et les instances régionales ou nationales. Néanmoins, l'ensemble de l'organisation se fixe comme objectif de faciliter l'insertion militante des mères. Ainsi, Isabelle Verhaeghe, première mère de famille à remplir la fonction de secrétaire générale du MPF-MLP ${ }^{14}$ au tournant des années 1950, exerçait ses responsabilités nationales en étant remplacée dans son rôle de mère. Elle avait obtenu en défraiement, et pour seul salaire, quatre journées hebdomadaires de travailleuses familiales payées par l'organisation. Cela lui faisait dire que son travail n'était pas reconnu, contrairement à celui des autres responsables nationaux, salariés.

Durant les années d'immédiat après-guerre, l'UFF, dans le cadre de la famille traditionnelle, met également en place des structures d'aide à la famille et aux mères : service social national, colonies de vacances, garderies, dispensaires, "foyer Danielle Casanova pour l'éducation de la femme ", dépistage de la tuberculose... En effet, est-il 
dit, « l'amélioration des conditions de vie, c'est une préoccupation constante de l'UFF depuis sa naissance » et alléger les problèmes journaliers donne confiance dans l'action collective, donne envie de la développer ${ }^{15}$. Mais cette aide concrète multiforme aux mères ne dure pas, sous une triple influence : la guerre froide politise l'action de l'UFF et entraîne une chute des effectifs; les services ne correspondent pas à sa volonté de regrouper les femmes autour de revendications présentées aux pouvoirs publics; enfin, les municipalités communistes mettent en place des structures d'aide, jouant, " mère sociale " suprême, le rôle assigné en URSS à l'État pour libérer les mères du travail domestique et éducatif. Cette conception du rôle fondamental de l'État dans le processus souhaité de transformation sociale n'est pas totalement celle du MPF, pour qui c'est d'abord aux familles et aux associations de remplir cette fonction. La réflexion et la pratique du MPF s'organisent en effet selon le principe de subsidiarité, conjugué avec le refus du paternalisme et la volonté d'une prise de distance vis-à-vis du Parti communiste ${ }^{16}$.

20 Notons aussi que le partage des tâches est évacué selon une logique différente de celle qui prévaut dans la mouvance MPF où l'on ne parle pas d'oppression, puisqu'on considère que la maternité accomplit totalement « la » femme. La défense de ses droits et des besoins de la famille lui incombe puisqu'elle est responsable de ce secteur dans sa globalité. C'est la société et le manque de moyens qui l'enchaînent. Une fois «libérée » - c'est-à-dire libérée de son trop plein de tâches physiques et maternantes -, elle est disponible pour son rôle de mère et d'épouse. Cela n'invalide pas la légitimité de son intervention militante dans l'espace public qui lui est accessible selon sa volonté quand les enfants lui en laissent la possibilité. Ce sésame moral entérine une relative disponibilité sur les créneaux horaires scolaires, mais ne souligne pas assez que le travail domestique et éducatif reste le lourd attribut des mères. En tout cas, celles-ci ne sont pas perçues comme des concurrentes par et pour les hommes, qui conservent leur fonction sociale de chef de famille, travailleur, pourvoyeur financier. Au moment où Maurice Thorez, en 1945, proclame qu' ' il n'est pas de libération véritable de la femme sans indépendance économique par son travail dans la production ", le Mouvement Républicain populaire (MRP), dont le MPF est encore plus ou moins proche, s'insurge contre les prises en charge collectives d'enfants et propose le travail à mi-temps pour les femmes ${ }^{17}$. C'est à la fin des années 1940 que la mouvance MPF commence, d'abord timidement, à proposer le travail aux femmes mariées, "si elles le souhaitent ». Et plusieurs décennies sont nécessaires dans certaines régions pour concrétiser cette ouverture dans l'ensemble de la mouvance.

Le genre du militantisme

21 Concrètement, la mouvance MPF propose un militantisme de couple où les responsabilités sont partagées selon un idéal de complémentarité considéré comme égalitaire, sur le plan des tâches familiales comme sur celui du militantisme. Le triangle père-mère-enfant $y$ fonde la famille ouvrière et la répartition des rôles en son sein. Aux hommes, pourvoyeurs financiers de la famille, reviennent le travail extérieur et l'engagement syndical, forme essentielle du militantisme ouvrier préconisé, même si l'investissement politique n'est pas, et sera de moins en moins, modeste. Aux mères, donc, est confié le travail au foyer, complètement légitimé et valorisé. Gardiennes du foyer, épouses et éducatrices, elles sont les garantes de la bonne marche de la famille. Le travail extérieur est reconnu pour les jeunes filles qui pourront en faire, si elles y sont attachées, le « sacrifice » une fois épouses et surtout mères. 
22 Les partages sexués ne sont pas réellement remis en question puisque l'épouse au foyer est responsable du front domestique, avec les charges qui lui incombent, et le mari du front extérieur. La notion de complémentarité est ici capitale. Dans le partage au niveau du couple, les femmes sont valorisées en tant que mères au foyer et recherchées en tant que militantes. À condition que le devoir maternel et ménager, conçu comme indispensable, soit parfaitement accompli, l'engagement social des mères devient non seulement possible mais nécessaire, pour l'accomplissement du couple et de son « devoir social ». Les femmes interviennent donc en tant que militantes sur ce qui, dans la sphère publique, les concerne, elles et les autres mères de la classe ouvrière (prix, politique familiale, logement, école, mais aussi loisirs...). Les tâches militantes masculines et féminines s'interpénètrent constam-ment, les femmes agissant sur le terrain politique considéré comme plus masculin en organisant des entrevues avec différents interlocuteurs: conseillers municipaux, députés, voire ministres. De leur côté, les hommes interviennent aux côtés des femmes pour obtenir l'adhésion d'une famille, pour faire circuler ou réparer les machines à laver collectives... Toutefois, le militantisme de quartier, pratiqué en « horaires de journée », est prioritairement celui des femmes.

23 Les objectifs de la mouvance MPF et de ses militantes, doubles mères sociales, sont honorablement remplis. Durant les Trente Glorieuses, la mise en place d'une aide concrète par les services a entraîné l'émergence de militantes de quartier ; l'axiome de l'auto-prise en charge du monde ouvrier a fait sortir des femmes-mères de chez elles, et la formation globale a contribué à les transformer, même modestement, en femmessujets. Toutefois des nuances doivent être apportées : l'auto-prise en charge est surtout vraie pour les responsables, la «base " suivant plus ou moins l'impulsion donnée. À Marseille, certains doutent même de l'existence d'une promotion autre qu'individuelle de quelques éléments ouvriers ${ }^{18}$. Par ailleurs, l'inégalité sexuée persiste, y compris dans le militantisme. Elle est sous-tendue par l'absence de l'idée même de partage des tâches, et par l'existence de deux terrains d'action (usine et quartier) dont la reconnaissance, la représentation et la valorisation sociales sont différentes et inégales. La revendication d'un syndicalisme de quartier n'empêche pas les femmes de percevoir cette hiérarchisation interne des militances. L'une d'elles évoque ainsi l'existence "d'une hiérarchie des valeurs : pour les militants, le syndicalisme de la production est le plus important $»^{19}$. Cette hiérarchisation est exprimée par les hommes à l'occasion et sans même le vouloir. Ainsi, pour Alphonse Garelli, ancien responsable national du MLP, ce n'est pas le quartier mais bien l'usine qui est le lieu de la prise de conscience ouvrière et l'endroit où il faut agir en prioritée ${ }^{20}$. Syndicalisme ouvrier, action politique restent un terrain privilégié pour les hommes, les femmes accompagnant les actions. Toutefois, les sections MLP et MLO réfléchissent en couple sur la question des guerres coloniales et tous vont aux manifestations, ce qui est peu répandu chez les militantes exclusivement liées aux services.

24 La maternité sociale, finalement, continue à se conjuguer essentiellement au féminin. C'est le "pré carré » militant des femmes. Et l'engagement des "mères sociales " suppose " que tout soit fait à la maison quand même $"^{21}$. Certes ces tâches sont valorisées par la mouvance MPF; certes, cela correspond à une certaine logique puisque le travail féminin au foyer (mais sans salaire...) est l'équivalent du travail masculin extérieur (mais salarié...). Mais surtout, remettre en cause le partage sexué du travail, c'est-à-dire l'ensemble du travail, interne et externe au foyer, n'entre pas dans 
sa conception. Le mouvement repose sur le couple "normal», ouvrier et chrétien, malgré l'acceptation et la valorisation de la place des femmes-mères. Les tâches domestiques sont allégées ou socialisées pour «libérer" les femmes, mais au MPF comme au PCF, un changement de répartition n'est pas vraiment pensé. La notion de services libérant les femmes-mères les enferme de facto dans leur "nature ", même si elle ouvre au militantisme, sorte de double journée pour les femmes au foyer. D'autre part, l'objectif d'aider les femmes, d'alléger « leurs » tâches domestiques et éducatives traduit aussi, y compris de la part de la mouvance MPF qui pourtant, de façon consciente, ne se situe pas du tout dans cette optique, une forme de condescendance, voire, à l'extrême, de dédain. En ce sens, la maternité sociale des organisations est le reflet d'un " père-social » dont les caractéristiques restent à délimiter et approfondir, paternalisme des hommes envers les femmes, envers les mères.

25 L'évolution de la société dans la décennie 1960 modifie le contexte d'exercice de la maternité sociale des militantes. Le développement des grandes surfaces et des laveries commerciales, la possibilité, pour un nombre croissant de familles populaires, d'acheter des appareils ménagers, sape la base même de fonctionnement des services. Ainsi, celui des machines à laver décline après l'apogée du milieu de la décennie. Certains services se maintiennent, mais ils ne sont plus aussi centraux dans l'activité et le recrutement de l'organisation. Par contre, des associations, nées elles aussi du MPF, se pérennisent et se professionnalisent, tout en conservant des éléments de l'orientation maternité sociale originelle. La logique de service perdure, même si c'est souvent dans le cadre d'une institutionnalisation. Il en est ainsi des services d'aide familiale, qui se sont autonomisés par rapport aux mouvements familiaux, de façon plus ou moins marquée selon le courant concerné, mais la logique d'intervention sociale y prend le pas sur l'objectif de promotion collective des mères.

26 Ainsi, la mouvance MPF a donné naissance à une maternité sociale caractéristique du deuxième tiers du XXe siècle. Adaptée à la société et à l'économie de ce temps, elle ne put résister à leurs transformations. Satisfaits par les développements de la consommation, transformés par les changements sociaux, les ménages ouvriers ne ressentaient plus le besoin d'adhérer par le biais des services. De plus, une nouvelle génération militante a fait pression pour un engagement sur les multiples fronts de la décennie 1970, qui ne résistèrent d'ailleurs pas aux modifications sociales, économiques et politiques de la décennie suivante. Les associations se recentrent alors sur des activités plus "classiques» que disent les changements de sigle successifs: l'APF devient CSCV (Confédération syndicale du cadre de vie) en 1976, puis CLCV (Consommation, logement, cadre de vie) en 1998.

27 Comme pour les philanthropes du XIX ${ }^{\mathrm{e}}$ siècle, l'un des paradoxes de cette maternité sociale militante a été, en faisant sortir les femmes mariées de chez elles et en leur faisant prendre des responsabilités, de contribuer à ce que certaines reconsidèrent la posture de mère au foyer. De nombreuses militantes, à partir des années 1970, sont personnellement concernées par le passage, dans l'ensemble de la société, de l'idéal de la mère au foyer au modèle du cumul des fonctions de mère et de travailleuse. De plus en plus salariées, et attachées à la reconnaissance sociale procurée par le salariat, elles sont moins disponibles que leurs aînées, et ne conçoivent plus la maternité - sociale et/ ou réelle - comme l'axe central d'une activité militante. Tout en conservant un investissement associatif, elles cherchent dans le travail salarié une liberté et une reconnaissance sociale que, d'après elles, le militantisme ne suffit plus à leur apporter. 
Toutefois, le contexte de crise économique des années 1980 remet à l'ordre du jour, en réponse aux besoins, des initiatives proches de la maternité sociale d'antan: groupements de commandes, entretien du linge, réseaux d'échange de savoir ${ }^{22}$.

\section{BIBLIOGRAPHIE}

Cova Anne, 1997, Maternité et droit des femmes en France, XIX ${ }^{e}-X X^{e}$ siècles, Anthropos historique, Anthropos, Paris.

Dermenjian Geneviève (dir.), 1991, «Femmes, famille et action ouvrière », Les Cahiers du GRMF n 6, 304 pages .

Les Cahiers du GRMF . Le GRMF - Groupement pour la recherche des Mouvements familiaux populaires -, 4 allée du Ternois, 59650 Villeneuve d'Asq, a fait paraître 13 cahiers sur l'histoire des Mouvements familiaux populaires. Le Cahier $n^{\circ} 13$ « Dans le combat ouvrier. Le mouvement populaire des familles dans les Bouches-du-Rhône ", 326 pages, dirigé par Geneviève Dermenjian est paru en 2004.

Dermenjian Geneviève et Fishman Sarah, 1996, «Les femmes de prisonniers de 1940 », in Vingtième siècle, revue d'histoire, $\mathrm{n}^{\circ}$ 49, janvier-mars 1996.

Dussuet Annie et Loiseau Dominique, « Mouvements familiaux entre logique de service et logique d'autogestion ", Autogestion, la dernière utopie ?, direction Frank Georgi, publications de la Sorbonne, 2003, pages 571-584.

Knibiehler Yvonne, 1991, Histoire des mères et de la maternité en occident, Paris, PUF, Que sais-je ? n 3539.

Loiseau Dominique, 1996, Femmes et militantismes, L'Harmattan, 240 pages.

Molina Claire, Les femmes à la libération, maîtrise d'histoire, Paris VII, 1977.

Perrot Michelle, 1991, « Sortir », in Histoire des femmes en Occident, tome IV, Plon, p. 487-494.

\section{NOTES}

1. D'ailleurs non obligatoirement exercée par des femmes. Toutefois, l'activité des organisations masculines et celles de l'Etat est laissée pour le moment de côté.

2. Perrot $1991: 468$.

3. Cf. Cova 1997 ; Knibiehler 2002 ; Perrot 1991.

4. Même si la Vierge l'incarne sous forme de «l'amour symbolique ».

5. Voir les encycliques Casti connubii de 1930 et Quadragesimmo anno de 1931.

6. Et de nombreuses autres associations, comme les travailleuses familiales, les maisons de vacances...

7. Cette seconde dimension ne sera pas traitée ici. Dermenjian 1991, Loiseau 1996. 
8. La promotion consiste à faire progresser les militants et le monde ouvrier pour les faire gagner en conscience ouvrière, les former de façon personnelle et citoyenne, les mener en somme de l'enfance à l'âge adulte.

9. L'Humanité, 28 juin 1947.

10. Remarquons que cet objectif a totalement disparu depuis, dans l'ensemble des professions chargées d'aider les mères de famille.

11. Dermenjian 1991. Dermenjian et Fishman 1996.

12. Fishman 1991.

13. Entretien avec Isabelle Brisset, militante MLO/APF, Loiseau 1996.

14. Dermenjian 1991.

15. Aperçu de l'activité UFF depuis sa naissance, document UFF 1982.

16. Bruno Duriez et Jean Nizey, « Les services du MPF. Entre apostolat et réponse à des besoins ", Cahiers du GRMF, n 11, 2002, p. 17-60.

17. Discours du 11/4/1945 et Conseil national du 4/7/1946, Molina 1977.

18. Entretien avec Cécile Bossy, Cahiers $n^{\circ} 13,2004$, p. 67-71.

19. Entretien avec Madeleine Juin, militante APF/CSCV.

20. Entretien avec Alphonse Garelli, Cahiers $n^{\circ} 13,2004$, p. 199-212.

21. Expression utilisée par de nombreuses militantes lors des entretiens.

22. Dussuet et Loiseau 2003.

\section{RÉSUMÉS}

L'Action catholique spécialisée a donné naissance pendant le deuxième tiers du $\mathrm{XX}^{\mathrm{e}}$ siècle au Mouvement populaire des familles (MPF) et à ses dérivés. Ces Mouvements se donnaient pour but la formation et la promotion du monde ouvrier par lui-même, notamment par le biais de la famille. Les mères de famille au foyer prenaient en charge les intérêts de leur quartier et de toutes les familles ouvrières, assumant ainsi une maternité sociale. Elles organisaient des délégations auprès des mairies, créaient et géraient des services (Femmes de prisonniers pendant la Seconde Guerre mondiale, services de machines à laver, travailleuses familiales pendant les trente glorieuses...). Le modèle d'action du MPF entre en déclin avec la modernisation du pays puis, dans les années 1970 avec l'accès généralisé des femmes au travail.

\section{AUTEURS}

\section{GENEVIÈVE DERMENJIAN}

Geneviève DERMENJIAN, maîtresse de conférences en histoire contemporaine à l'IUFM d'AixMarseille (HDR) et membre de l'UMR Telemme travaille sur l'histoire des femmes, des jeunes et de la famille, en particulier dans la JOC et dans les Mouvements familiaux populaires issus de la JOC. Parmi les publications récentes : coordination avec Mathilde Dubesset de « Chrétiennes , $\mathrm{n}^{\circ} 6$ de Clio, Histoire, Femmes et sociétés (2002) ; codirection avec Jacques Guilhaumou et Martine Lapied de Le Panthéon des femmes; Pratiques et représentations des héroïnes (Publisud, 2004) ; « Les 
associations de femmes au foyer : des occupations différenciées de la rue », en collaboration avec Dominique Loiseau, in Les femmes et la Ville, Syvette Denèfle (dir.), Tours, 2004.

\section{DOMINIQUE LOISEAU}

Dominique LOISEAU, enseignante dans le secondaire, membre du LESTAMP de l'Université de Nantes, travaille sur les rapports sociaux de sexe à l'oeuvre dans le militantisme, en particulier sur le plan du syndicalisme, des associations de quartier et du parti communiste. Parmi les publications récentes : «Mouvements familiaux entre logique de service et logique d'autogestion, 1945-2000 ", avec Annie Dussuet, in Autogestion, la dernière utopie ? F. Georgi (dir.), Publications de la Sorbonne, 2003 ; «Les (343) 'salopes' et les ménagères. Paroles de femmes et classes sociales », in Paroles de femmes, coordonné par F. Le Jeune, CRINI, Nantes, 2003 ; « Noir de femmes :

conformités et transgressions ", in Les oeuvres noires de l'art et de la littérature, A. Pessin et M.-C. Vanbremeersch (dir.), L'Harmattan, 2002 